\title{
Acoustic source localization in an anisotropic plate without knowing its material properties: a new approach
}

\author{
Won Hyun Park ${ }^{\mathrm{a}}$, Pawel Packo ${ }^{\mathrm{b}}$ and Tribikram Kundu ${ }^{\mathrm{a}, \mathrm{c}}$ \\ ${ }^{a}$ Dept. of Aerospace and Mechanical Engineering, University of Arizona, Tucson, AZ 85719, U.S.A; \\ ${ }^{\mathrm{b}}$ AGH University of Science and Technology, Krakow, Poland; \\ ${ }^{\mathrm{c}}$ Dept. of Civil Engineering and Engineering Mechanics, University of Arizona, Tucson, AZ 85719, \\ U.S.A
}

\begin{abstract}
A number of techniques are available for acoustic source localization in isotropic plates without knowing the material properties of the plate. However, for a highly anisotropic plate acoustic source localization requires some knowledge of the plate material properties or its group velocity profile. In absence of this information one requires a large number of sensors to predict the acoustic source point in the plate. All proposed techniques for acoustic source localization with a few sensors assume the straight line propagation of waves from the source to the receiving sensor with an average group velocity when the plate material properties are not known. However, this assumption is not true for an anisotropic plate. Although the currently available techniques work well for weakly anisotropic plates since the wave path does not deviate significantly from the straight line propagation they fail miserably for highly anisotropic plates.

In this paper acoustic source is localized in an anisotropic plate when non-circular wave front is generated. Direction vectors of wave fronts are obtained from the Time-Difference-Of-Arrivals (TDOA) at three sensors placed in a cluster. Four such direction vectors are then utilized in geometric vector analysis to accurately obtain the acoustic source location. The proposed technique is illustrated on an orthotropic plate that generates rhombus shaped wave front. It should be noted that the proposed technique does not require wave propagation along a straight line from the source to the sensor. It also does not need the knowledge of the material properties of the plate.
\end{abstract}

\section{INTRODUCTION}

Various techniques for acoustic source localization have been reviewed by Kundu [1]. Different versions of the acoustic source localization technique in an anisotropic plate with known velocity profile are available in the literature (Kundu et al. [2-4], Hajzargerbashi et al. [5], Koabaz et al. [6], Nakatani et al. [7]). Most of these techniques require optimization scheme such as simplex algorithm [8] and genetic algorithm [9-10].

Kundu [11] proposed a new technique by which an acoustic source could be approximately localized in an anisotropic plate with the help of six receiving sensors. This technique neither needed the knowledge of the plate properties such as the direction dependent velocity profile in the plate nor required to solve a system of nonlinear equations. Kundu et al. [12] experimentally verified the technique for plates made of both isotropic (requiring 4 sensors) and anisotropic materials (requiring 6 sensors). Ciampa et al. $[13,14]$ also proposed a technique for source localization in anisotropic plates without knowing the plate properties. However, their technique required the solution of a system of nonlinear equations.

Baxter et al. [15] and Xiao et al. [16] also proposed techniques for acoustic source localization when the structural properties are unknowns. The technique proposed by Baxter et al. [15] required initial training of the structure by a system of known acoustic sources at different locations and then that knowledge was used for localizing the future acoustic events by comparing the recorded arrival time difference (that they called Delta-T) between different sensor pairs with those from the training dataset. For large structures the training part can be very time consuming. Xiao et al. [16] used two arrays of sensor in mutually perpendicular directions ( $\mathrm{x}$ and $\mathrm{y}$ ) and localized acoustic source from these two arrays by beam forming method. Since for beam forming method the localization accuracy is not very high in the

Health Monitoring of Structural and Biological Systems 2016, edited by Tribikram Kundu,

Proc. of SPIE Vol. 9805, 98050J · @ 2016 SPIE · CCC code: 0277-786X/16/\$18 · doi: 10.1117/12.2224504 
array direction the authors showed that by considering two perpendicular arrays the acoustic source can be localized with reasonably high accuracy in a plate like structure even when there is uncertainty about the propagating wave velocity.

Recently Kundu et al. [17] proposed a hybrid technique by estimating initial source coordinates and then minimizing appropriate objective function to accurately find the source location. The hybrid technique converges faster than the method proposed by Ciampa et al. $[13,14]$ that did not have any way of generating first estimates of the unknown parameters. However, the hybrid technique still had some prediction error because the basic assumption that the wave propagates from the acoustic source in different directions with the direction dependent average group velocity values is not true. In this paper we propose a new technique to overcome the existing shortcomings by changing the analysis perspective from the angular dependent group velocity of the wave and its straight line propagation to specific wave front shape and its geometric properties.

\section{DIRECTION VECTORS MEASURED BY SENSOR CLUSTER}

Kundu et al. $[11,12]$ introduced a simple right-angle configuration of three sensors where two sensors are placed orthogonally at the same distance $(d)$ from one sensor as shown in Figure 1. The three-sensor configuration is called a 'Sensor cluster'. The cluster is mounted on the surface of the plate. A couple of clusters acquire wave signals synchronously. Assume that the distance $d$ between the sensors in a cluster is much smaller than the distance from the source location to the cluster. Then the wave front passing through the cluster can be considered as a plane wave front as depicted in Figure 1.

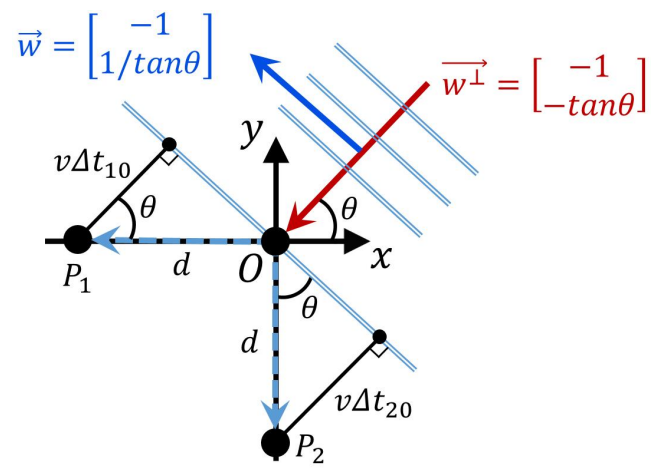

Figure 1 Wave front is considered as flat or a plane wave front at the sensor cluster location. Sensors $P_{1}$ and $P_{2}$ are located at a distance d from Sensor $O$. Direction vector $\left(\overrightarrow{w^{\perp}}\right)$ and parallel vector $(\vec{w})$ of the plane wave front are obtained by measuring TDOAs between two sensors and computing their ratio, $\tan \theta=\Delta t_{20} / \Delta t_{10}$.

The direction vector $\left(\overrightarrow{w^{\perp}}\right)$ and the parallel vector $(\vec{w})$ of the plane wave front can be described as

$$
\left[\overrightarrow{w^{\perp}} \vec{w}\right]=\left[\begin{array}{cc}
-1 & -1 \\
-\tan \theta & 1 / \tan \theta
\end{array}\right]
$$

where $\tan \theta$ is the ratio of two TDOAs

$$
\tan \theta=\frac{\sin \theta}{\cos \theta}=\frac{v \Delta t_{20} / d}{v \Delta t_{10} / d}=\frac{\Delta t_{20}}{\Delta t_{10}}=\frac{t_{2}-t_{0}}{t_{1}-t_{0}}
$$

With the plane wave front approximation, one can assume that the three sensors receive identical signal patterns at the first arrival and the signals are dispersed afterwards. TDOA is a time shift between two received signals which is computed by the cross-correlation technique by plotting the product of two signals when for one signal the time shift is continuously changed. Two signals recorded by two sensors are displayed together in Figure 2(a). Almost identical signals with a small time shift are found in the range between $1100 \mu \mathrm{s}$ and $1500 \mu \mathrm{s}$. The time shift between them is clearly found. 
In order to obtain TDOA, cross-correlation technique is adopted. This method examines the similarity between the two given signals:

$$
[F(t) \star G(t)](\tau)=\int_{\text {lower bound }}^{\text {upper bound }} F(t) G(t+\tau) d t
$$

The maximum value of the cross-correlation plot corresponds to the time shift or TDOA difference as shown in Figure 2(b).

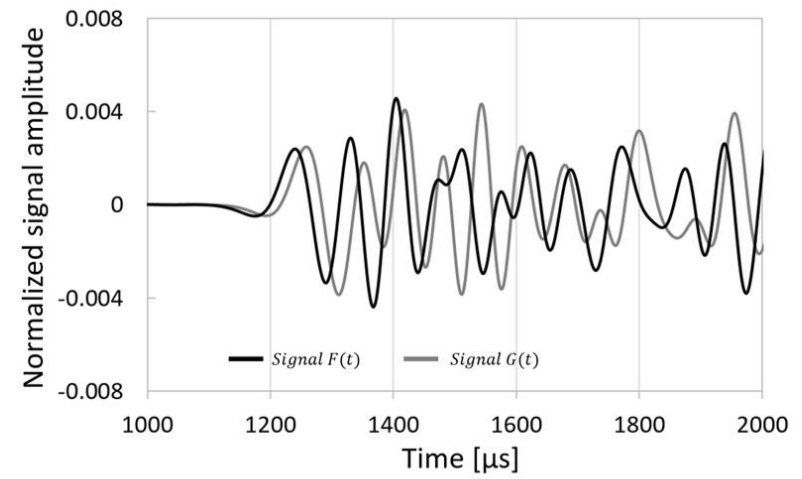

(a)

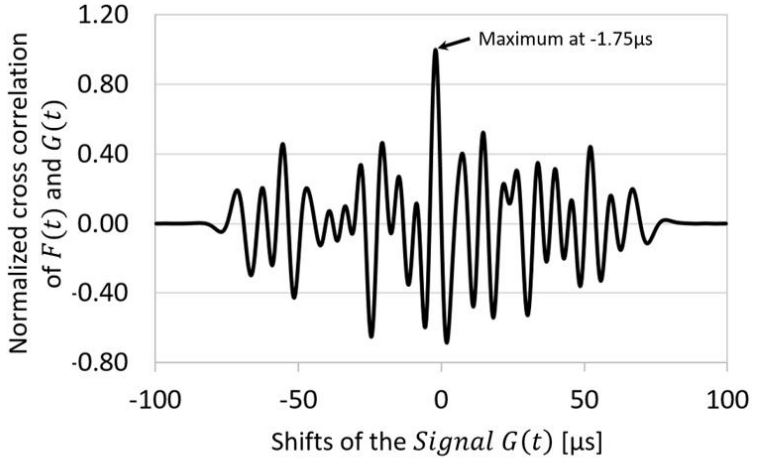

(b)

Figure 2 (a) Signal $F(t)$ is measured at sensor $O$, and signal $G(t)$ is measured at sensor $P_{1}$. Note that the signal patterns are very similar in the time range $1100 \mu \mathrm{s}$ to $1500 \mu \mathrm{s}$. (b) Cross-correlation between the two signals is computed and displayed. Maximum correlation exist at $-1.75 \mu \mathrm{s}$ which corresponds to the time shift between the two signals.

\section{NUMERICAL SIMULATION OF WAVE PROPAGATION IN A THIN ANISOTROPIC PLATE}

A $500 \mathrm{~mm} \times 500 \mathrm{~mm} \times 2 \mathrm{~mm}$ thin anisotropic plate is modeled by cuLISA3D software for numerical simulation with orthotropic plate material properties as follows:

- Mass density : $1.5 \times 10^{-9}$ tonnes $/ \mathrm{mm}^{3}=1.5 \mathrm{gm} / \mathrm{cc}$

- $\quad$ Elastic moduli : $E_{1}=66400 \mathrm{MPa}, E_{2}=6000 \mathrm{MPa}$, and $E_{3}=6000 \mathrm{MPa}$

- Poisson's ratios : $v_{12}=0.2, v_{23}=0.25$, and $v_{31}=0.25$

- Shear moduli : $\mathrm{G}_{12}=1400 \mathrm{MPa}, G_{23}=2100 \mathrm{MPa}$, and $G_{31}=2100 \mathrm{MPa}$

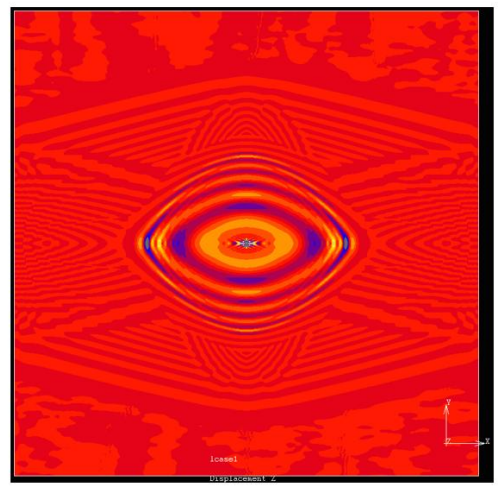

(a)

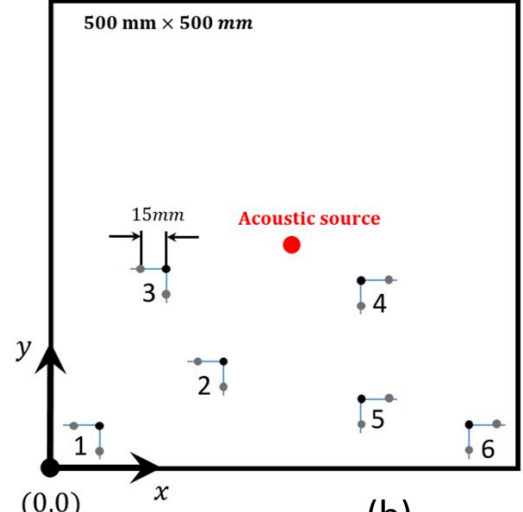

(b)

Figure 3 (a) In the numerical simulation an acoustic source generates various wave fronts from the acoustic source. This plot is obtained at $t=100 \mu \mathrm{s}$. (b) 6 clusters at different locations record the wave signal. Only 4 clusters (as shown later in Figure 9) are needed to localize the acoustic source. Sensors in each cluster are $15 \mathrm{~mm}$ apart along both horizontal and vertical directions. For example, three sensors of sensor cluster 1 are located at (35,50), (50,50), and (50,35). 
Acoustic source is located at the center of the plate $(250,250)$ and excited by a two-period sine signal modulated by Gauss window identified from an artificial source signal (pencil lead break) in an inverse procedure. Free boundary conditions are used in the model. Figure 3(a) shows resultant wave fronts of the numerical simulation at $\mathrm{t}=100 \mu \mathrm{s}$. Different sensor-clusters can be placed at various positions as shown in Figure 3(b). Sensors in each cluster are $15 \mathrm{~mm}$ apart in both horizontal and vertical directions. Time histories recorded by the sensors can be obtained from the numerically simulated data, as shown in Figures 2 and 4.

All 18 sensors monitor incoming signals and store them until the simulation ends. One received signal (dark black solid line) is plotted in Figure 4. When we take absolute value of the signal and plot it in logarithmic scale then three different magnitude levels are revealed. The lowest level indicates the white noise of the simulated data. First wave group hits the sensor carrying relatively small energy but propagating faster while second wave group arrives later with higher level of energy (see gray solid line in Figure 4).

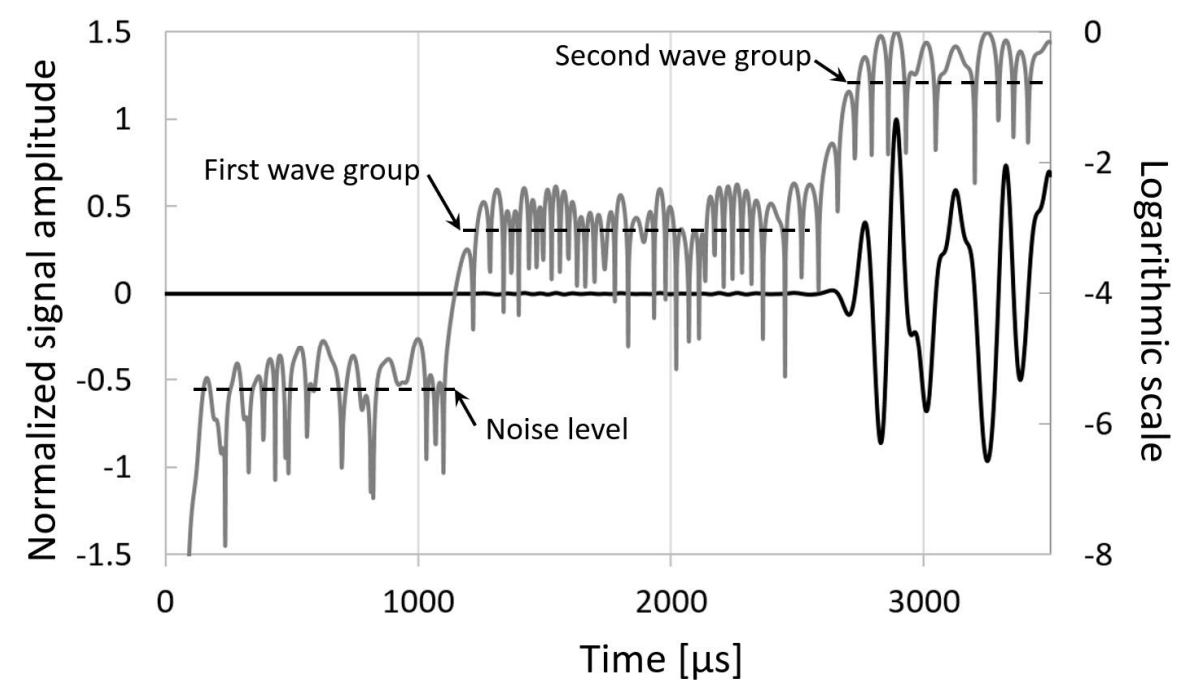

Figure 4 A signal recorded by one sensor is plotted in dark black solid line. When absolute value of the signal is plotted in logarithmic scale as shown in gray solid line, three significantly different levels of signal energy are noticed which are white noise, first wave group arrival and second wave group arrival, respectively. 


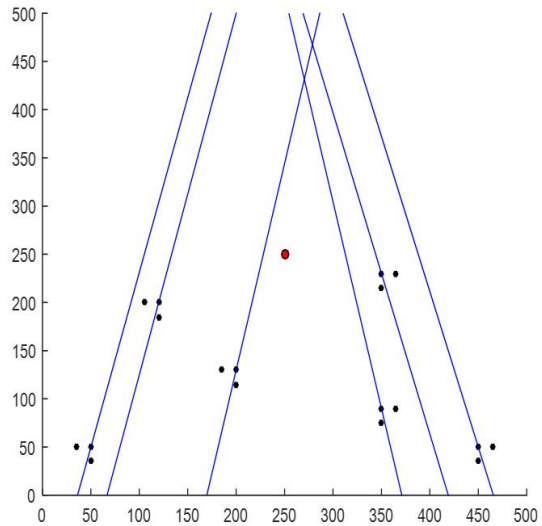

(a)

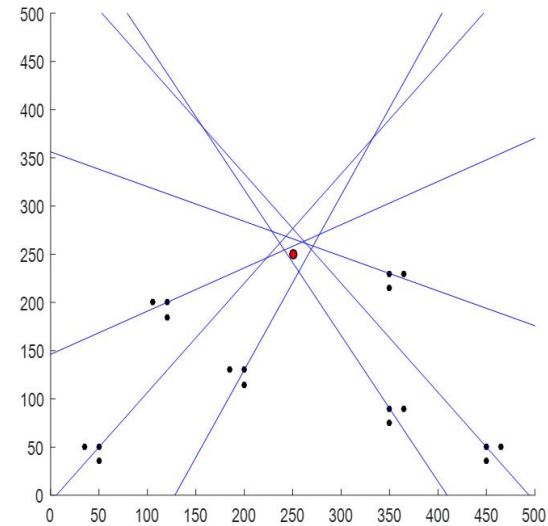

(b)

Figure 5 Lines parallel to each $\overrightarrow{w^{\perp}}$ are displayed where $\overrightarrow{w^{\perp}}$ are obtained from measured TDOAs at each cluster. (a) First wave group: rhombus wave front, (b) Second wave group: non-circular wave front.

All $\overrightarrow{w^{\perp}}$ described in section 2 are computed by measuring TDOAs at each cluster for first and second wave groups. Then lines along each $\overrightarrow{w^{\perp}}$ are drawn in Figure 5. Kundu [1] indicated that the lines should go through the acoustic source if the wave front is circular. For non-circular wave fronts, they do not go through a common point as in Figure 5. Especially in Figure 5(a), almost two sets of parallel lines are observed on two sides of the acoustic source location. One possible wave front that can produce such wave propagation direction is rhombus. In the next section, the new source localization technique is discussed for rhombus wave fronts.

\section{PROPOSED NEW TECHNIQUE FOR RHOMBUS WAVE FRONT}

Propagating waves form non-circular wave front in anisotropic plates. Rhombus wave front can be a candidate when measured wave propagation direction vectors $\left(\overrightarrow{\mathrm{w}^{\perp}}\right)$ for two adjacent clusters are parallel regardless of the cluster's location as in Figure 5(a). In this example the rhombus wave front propagates faster than the other wave front so the sensors detect it first without any interference from the boundary reflected waves.

For acoustic source localization the concentric rhombus wave fronts depicted in Figure 6 are analyzed instead of finding the intersection point of a set of straight lines from sensor clusters. In other words, geometric properties of the concentric rhombus are utilized to determine the acoustic source location. All concentric rhombuses share a vertical diagonal and a horizontal diagonal, and the intersection of the two diagonals is the Acoustic Source location as shown in Figure 6. There are two basic requirements for this method. First, shape velocity of the rhombus wave front is constant, and two sensor clusters, for example $S_{r}$ and $S_{I}$, are placed such that the shape velocity can be obtained from these two clusters. The distance between the two sensor clusters $S_{r}$ and $S_{I}$ along the wave propagation direction is denoted by $d_{r 1}$. TDOA between these two sensor clusters is $\left(t_{r}-t_{1}\right)$. Then the shape velocity $\mu=\frac{d_{r 1}}{\left(t_{r}-t_{1}\right)}$. 


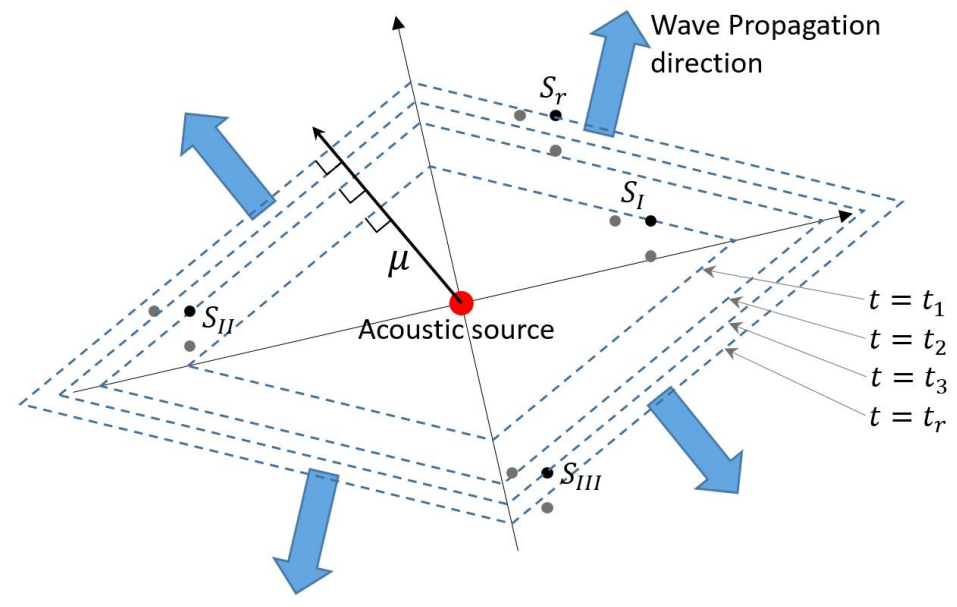

Figure 6 Rhombus wave front is generated by an acoustic source and expands with a shape velocity $\mu$. At $t=t_{1}$, sensor cluster $S_{I}$ captures the wave front first. Then cluster $S_{I I}, S_{I I I}$, and $S_{r}$ receive signals sequentially at $t_{2}, t_{3}$, and $t_{r}$, respectively. Simple vector analyses with direction vectors at all clusters and TDOAs allow to form the diagonals of the concentric rhombuses. The intersection point of the two diagonals gives the final acoustic source location.

In order to compute the shape velocity, the two clusters $S_{r}$ and $S_{I}$ must be located in the same quadrant; in other words, one plane wave front must pass through these two sensor clusters. Minimum number of required sensor clusters is 4 for localizing the acoustic source in an anisotropic plate. Vertical diagonal of the rhombus can be obtained by simple vector analysis with direction vectors and TDOA $\left(t_{2}-t_{1}\right)$ between clusters $S_{I}$ and $S_{I I}$. Similarly, horizontal diagonal of the rhombus can be obtained from direction vectors and TDOA $\left(t_{3}-t_{1}\right)$ between clusters $S_{I}$ and $S_{I I I}$. Detail derivation is given later. After two diagonals are obtained from the concentric rhombuses, the acoustic source location is identified from the intersecting point of the diagonals. Thus only geometric properties of the rhombus shape and direction vector measurements at four cluster positions are used to predict the exact source location without knowing any material properties or angular velocity profile of the anisotropic plate.

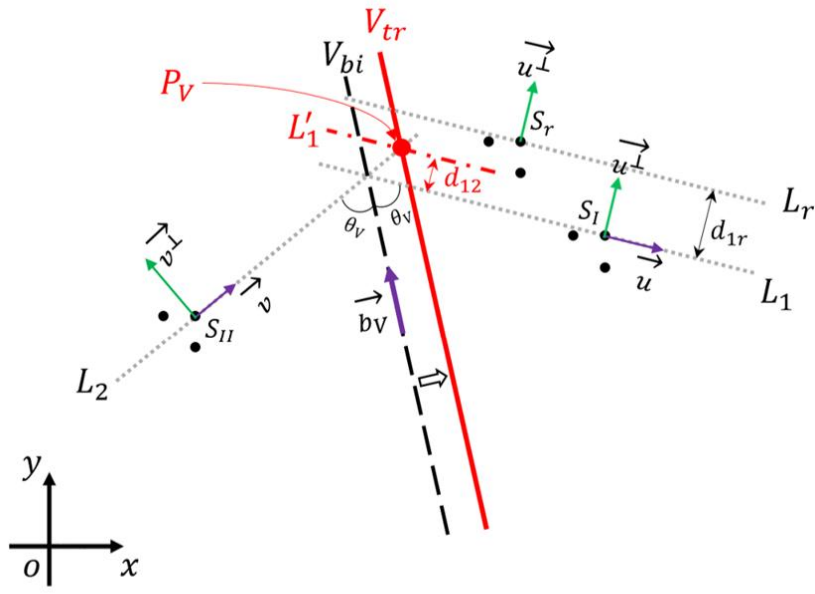

(a)

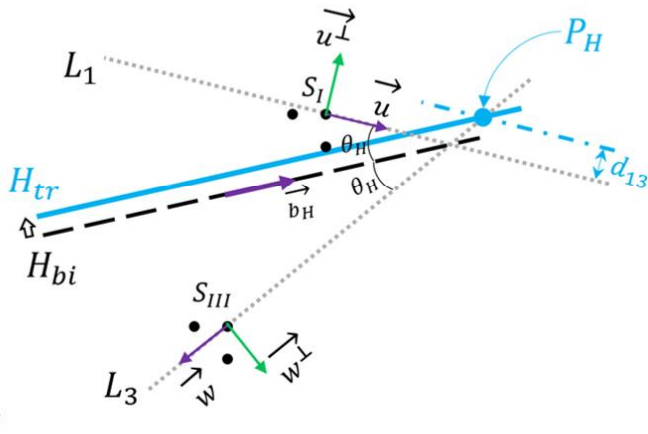

(b)

Figure 7 (a) $V_{t r}$ and (b) $H_{t r}$, are obtained from direction vectors and TDOAs at four clusters.

Remind that direction vectors and parallel vectors at each sensor cluster were discussed in section 2. Also crosscorrelation method for getting TDOAs between any two sensors was introduced. Vector analysis is started by estimating 
the shape velocity as given by Equation (4). Let us denote the direction vector at $S_{r}$ and $S_{I}$ as $\overrightarrow{u^{\perp}}$ and the parallel vector at $S_{I}$ as $\vec{u}$. The distance between wave front $L_{1}$ and $L_{r}$ is $d_{1 r}$, then the shape velocity can be obtained in the following manner:

$$
\mu=\frac{d_{1 r}}{t_{r}-t_{1}}=\frac{\left\|\operatorname{proj}_{\overrightarrow{u^{\perp}}}\left(\overrightarrow{S_{R}}-\overrightarrow{S_{I}}\right)\right\|}{t_{r}-t_{1}}=\left\|\frac{\left(\overrightarrow{S_{R}}-\overrightarrow{S_{I}}\right) \cdot \overrightarrow{u^{\perp}}}{\overrightarrow{u^{\perp}} \cdot \overrightarrow{u^{\perp}}}\right\| /\left(t_{r}-t_{1}\right)
$$

Another direction vector and parallel vector at $S_{I I}$ are denoted as $\overrightarrow{v^{\perp}}$ and $\vec{v}$, respectively. Next we introduce parametric representation for line $L_{1}$ on $2 \mathrm{D}$ plane, it is given as

$$
L_{1}=\left\{\overrightarrow{S_{I}}+\kappa_{1} \vec{u} \mid \kappa_{1} \in \mathbb{R}\right\}
$$

where $\mathbb{R}$ is real number space and all vector has two coordinate components, $(x, y)^{\mathrm{T}}$. If TDOA between $S_{I}$ and $S_{I I}$ is zero, the bisector $V_{b i}$ in Figure 7 becomes the vertical diagonal of the rhombus directly. Otherwise, the line $L_{1}$ should be shifted by $d_{12}$ to align the rhombus at $t=t_{2}$ in order to find the true vertical bisector, $V_{t r}$. Using the given shape velocity, the shifts is simply

$$
d_{12}=\mu\left(t_{2}-t_{1}\right)
$$

The shifted line $\left(L_{1}^{\prime}\right)$ may be derived from the following parametric representations involving the shift:

$$
L_{1}^{\prime}=\left\{\overrightarrow{S_{I}}+d_{12} \frac{\overrightarrow{u^{\perp}}}{\left\|\overrightarrow{u^{\perp}}\right\|}+\kappa_{1}^{\prime} \vec{u} \mid \kappa_{1}^{\prime} \in \mathbb{R}\right\}
$$

And the line $\left(L_{2}\right)$ passing through $S_{I I}$ is given in the same manner,

$$
L_{2}=\left\{\overrightarrow{S_{I I}}+\kappa_{2} \vec{v} \mid \kappa_{2} \in \mathbb{R}\right\}
$$

Now we solve $L_{1}^{\prime}=L_{2}$ to find the intersection $\left(P_{V}\right)$ of $L_{1}^{\prime}$ and $L_{2}$, denote that

$$
\overrightarrow{S_{I}}+d_{12} \frac{\overrightarrow{u^{\perp}}}{\left\|\overrightarrow{u^{\perp}}\right\|}+\kappa_{1}^{\prime} \vec{u}=\overrightarrow{S_{I I}}+\kappa_{2} \vec{v}
$$

By rearranging Equation 9 as follows, we may solve the simultaneous equations of two unknowns, $\kappa_{1}^{\prime}$ and $\kappa_{2}$ :

$$
\left[\begin{array}{l}
\kappa_{1}^{\prime} \\
\kappa_{2}
\end{array}\right]=\left[\begin{array}{ll}
\underline{u} & \underline{v}
\end{array}\right]^{-1}\left(\underline{S_{I I}}-\underline{S_{I}}-d_{12} \frac{u^{\perp}}{\left\|\underline{u^{\perp}}\right\|}\right)
$$

$P_{V}$ calculated by either substituting $\kappa_{1}^{\prime}$ into equation (7) or substituting $\kappa_{2}$ into equation (8) is used in the parametric formulation of the true bisector $V_{t r}$ (red solid line in Figure 7),

$$
V_{t r}=\left\{\overrightarrow{P_{V}}+\kappa_{V} \overrightarrow{b_{V}} \mid \kappa_{V} \in \mathbb{R}\right\}
$$

where $\overrightarrow{b_{V}}$ is parallel to the bisector and is obtained by adding two unit vectors:

$$
\overrightarrow{b_{V}}=\frac{\overrightarrow{u^{\perp}}}{\left\|\overrightarrow{u^{\perp}}\right\|}+\frac{\overrightarrow{v^{\perp}}}{\left\|\overrightarrow{v^{\perp}}\right\|}
$$

Obtaining the true horizontal bisector $\left(H_{t r}\right)$ as horizontal diagonal of the rhombus wave front follows exactly the same procedure as described in equations (6) to (12). We can have the new shift $\left(d_{13}\right)$ and the new shifted line $\left(L_{1}^{\prime \prime}\right)$ based on the TDOAs between $S_{1}$ and $S_{3},\left(L_{1}^{\prime \prime}\right.$ is shown later in Figure 9) 


$$
d_{13}=\mu\left(t_{3}-t_{1}\right)
$$

and

$$
L_{1}^{\prime \prime}=\left\{\overrightarrow{S_{I}}+d_{13} \frac{\overrightarrow{u^{\perp}}}{\left\|\overrightarrow{u^{\perp}}\right\|}+\kappa_{1}^{\prime \prime} \vec{u} \mid \kappa_{1}^{\prime \prime} \in \mathbb{R}\right\}
$$

The other line passing on $S_{I I I}$ is expressed with its direction vector $(\vec{w})$ as

$$
L_{3}=\left\{\overrightarrow{S_{I I I}}+\kappa_{3} \vec{w} \mid \kappa_{3} \in \mathbb{R}\right\}
$$

For the intersection $P_{H}$, we solve that

$$
L_{1}^{\prime \prime}=L_{3}
$$

and denote equation (16) that

$$
\overrightarrow{S_{I}}+d_{13} \frac{\overrightarrow{u^{\perp}}}{\| \overrightarrow{u^{\perp} \|}}+\kappa_{1}^{\prime \prime} \vec{u}=\overrightarrow{S_{I I I}}+\kappa_{3} \vec{w}
$$

By rearranging Equation (17) one gets the following system of simultaneous equations from which two unknowns, $\kappa_{1}^{\prime \prime}$ and $\kappa_{3}$ can be solved:

$$
\left[\begin{array}{l}
\kappa_{1}^{\prime \prime} \\
\kappa_{3}
\end{array}\right]=\left[\begin{array}{ll}
\underline{u} & \underline{w}
\end{array}\right]^{-1}\left(\underline{S_{I I I}}-\underline{S_{I}}-d_{13} \frac{\underline{u^{\perp}}}{\| \underline{u^{\perp} \|}}\right)
$$

$P_{H}$ calculated by either substituting $\kappa_{1}^{\prime \prime}$ into equation (14) or substituting $\kappa_{3}$ into equation (15) is used in the parametric formulation of the bisector $H_{t r}$ (blue solid line in Figure 8),

$$
H_{t r}=\left\{\overrightarrow{P_{H}}+\kappa_{H} \overrightarrow{b_{H}} \mid \kappa_{H} \in \mathbb{R}\right\}
$$

where $\overrightarrow{b_{H}}$ is parallel to the bisector and simple yield by adding two unit vectors:

$$
\overrightarrow{b_{H}}=\frac{\overrightarrow{u^{\perp}}}{\left\|\overrightarrow{u^{\perp}}\right\|}+\frac{\overrightarrow{w^{\perp}}}{\left\|\overrightarrow{w^{\perp}}\right\|}
$$




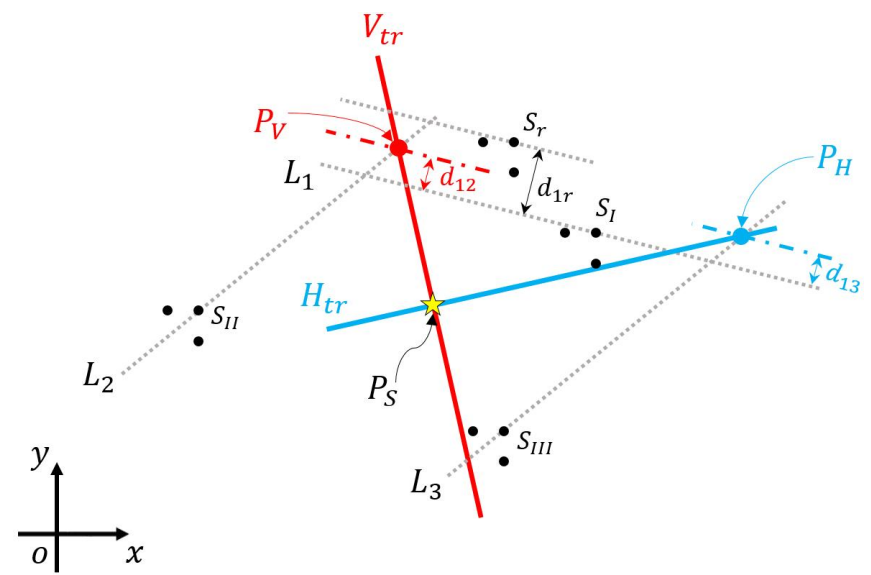

Figure 8 Two computed bisectors $\left(V_{r}\right.$ and $H_{t r}$ ) are drawn together. The intersection of two lines (star symbol) indicates the final acoustic source emission position obtained from a series of vector analysis with direction vectors and TDOAs.

Since the two true bisectors are diagonals of the rhombus wave front, we can conclude that the final intersection of $V_{t r}$ and $H_{t r}$ must be the acoustic source location. The final step to examine the source position $\left(P_{S}\right)$ is to solve the following relation

$$
V_{t r}=H_{t r}
$$

After substituting Equation (11) and Equation (19) into Equation (21) we get

$$
\overrightarrow{P_{V}}+\kappa_{V} \overrightarrow{b_{V}}=\overrightarrow{P_{H}}+\kappa_{H} \overrightarrow{b_{H}}
$$

After algebraic manipulation one gets,

$$
\left[\begin{array}{l}
\kappa_{V} \\
\kappa_{H}
\end{array}\right]=\left[\begin{array}{ll}
\underline{b_{V}} & \underline{b_{H}}
\end{array}\right]^{-1}\left(\underline{P_{H}}-\underline{P_{V}}\right)
$$

Estimated acoustic source location $\left(P_{S}^{*}\right)$ is finally determined by either substituting $\kappa_{V}$ into equation (11) or substituting $\kappa_{\mathrm{H}}$ into equation (19).

\section{NUMERICAL VALIDATION OF THE PROPOSED TECHNIQUE}

From the computer generated wave fronts shown in Figure 3 the acoustic source location is predicted using the proposed new technique. Figure 9 shows the positions of the four sensor clusters used to predict the acoustic source location in this geometry. Four clusters must be placed in three different quadrants. Note that the distance between the predicted point from the actual acoustic source location is only $2.68 \mathrm{~mm}$, while the distances between the source and four clusters vary from 130 to $283 \mathrm{~mm}$. 


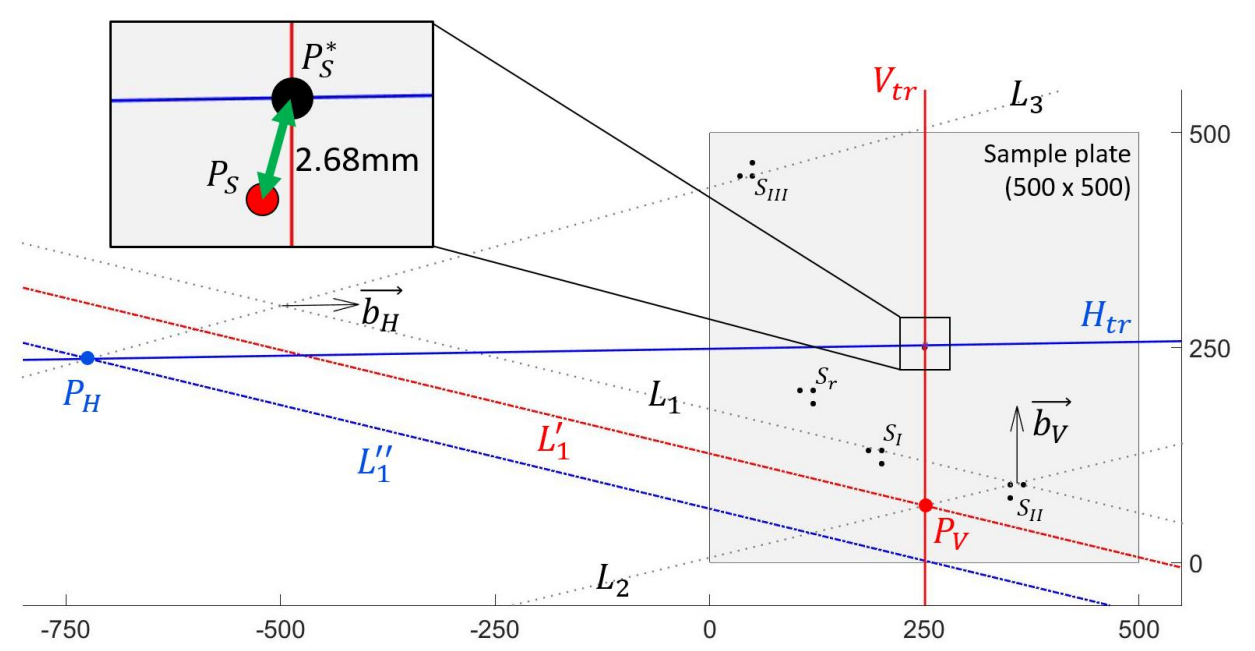

Figure 9 Computed lines, vectors and points from the numerical simulation and the vector analysis are drawn. The estimated location $\left(P_{S}^{*}\right)$ of the acoustic source is $(250.714,252.581)$ which is only $2.68 \mathrm{~mm}$ apart from the true location $\left(P_{S}\right)$.

Table 1 Computed vectors and points from the numerical simulation and the vector analysis are tabulated. Note that presented vectors are not normalized.

\begin{tabular}{|c|c|c|c|c|}
\hline & Coordinates $[\mathrm{mm}]$ & Computed vectors & & Computed vectors or points \\
\hline$S_{I}$ & $(200,130)$ & $\overrightarrow{u^{\perp}}=\left(\begin{array}{ll}-1 & -4.1429\end{array}\right)^{\mathrm{T}}$ & $\overrightarrow{b_{V}}$ & $\left(\begin{array}{ll}0 & -1.9442\end{array}\right)^{\mathrm{T}}$ \\
\hline$S_{I I}$ & $(350,90)$ & $\overrightarrow{v^{\perp}}=\left(\begin{array}{ll}1 & -4.1429\end{array}\right)^{\mathrm{T}}$ & $\overrightarrow{b_{H}}$ & $\left(\begin{array}{ll}-0.5006 & -0.0081)^{\mathrm{T}}\end{array}\right.$ \\
\hline$S_{I I I}$ & $(50,450)$ & $\overrightarrow{w^{\perp}}=\left(\begin{array}{ll}-1 & 3.6250\end{array}\right)^{\mathrm{T}}$ & $P_{V}$ & $(250.714,66.034)$ \\
\hline$S_{r}$ & $(120,200)$ & $\overrightarrow{u^{\perp}}=\left(\begin{array}{ll}-1 & -4.1429\end{array}\right)^{\mathrm{T}}$ & $P_{H}$ & $(-722.667,236.851)$ \\
\hline \multicolumn{3}{|c|}{ Rhombus shape velocity } & $\mu$ & $2.0112 \mathrm{~km} / \mathrm{s}$ \\
\hline \multicolumn{3}{|c|}{ Final estimation of acoustic source } & $\mathbf{P}_{\mathbf{S}}^{*}$ & $(250.714,252.581)$ \\
\hline
\end{tabular}

The analysis is started from calculating direction vectors, $\overrightarrow{u^{\perp}}, \overrightarrow{v^{\perp}}$ and $\overrightarrow{w^{\perp}}$ at each three-sensor cluster. The rhombus shape velocity $(\mu)$ is obtained from $\overrightarrow{u^{\perp}}$, TDOA $t_{r 1}$ between $S_{r}$ and $S_{I}$. TDOA $t_{21}$ and $\overrightarrow{u^{\perp}}$ allow to define the first shifted line $\left(L_{1}^{\prime}\right)$, then $P_{V}$ is the intersection between $L_{1}^{\prime}$ and $L_{2}$. Now the vertical diagonal (red solid line in Figure 9) of the rhombus wave front can be expressed with $P_{v}$ and the bisecting vector $\overrightarrow{b_{V}}$. In similar manner with two sensors, $S_{I}$ and $S_{I I I}$, the horizontal diagonal (blue solid line in Figure 9) can be obtained. Finally, the intersecting point by two diagonals is the estimated acoustic source location with only knowing coordinates of four clusters and measuring direction vectors. All computed vectors and points from the numerical simulation and the vector analysis are listed in Table 1.

\section{CONCLUSION}

A new technique for accurately predicting the acoustic source (such as an impact point) on an anisotropic plate, without knowing its material properties is introduced. This technique concentrates on geometric shape of the wave front and its geometric properties to exclude the previous shortcomings caused by utilizing the angular dependent group velocity of the wave or assuming straight line propagation of waves. As a result, it avoids the necessity of knowing material properties such as density, orthotropic elastic moduli and Poisson's ratios as long as two basic requirements are met which are: (1) at least twelve sensors in four clusters are used and (2) they are placed in three different quadrants. Since waves do not propagate along a straight line in anisotropic media all existing methods that assume straight line 
propagation of waves from the source to the sensor fail for anisotropic plates. In the current method this difficulty is overcome by utilizing the wave front shape for source localization instead of the propagation path from the source to the sensor. The technique is demonstrated on an orthotropic plate for which the wave front has a rhombus shape. The proposed technique correctly predicted the acoustic source position in this plate from the computer generated wave propagation data.

\section{References:}

[1] T. Kundu, "Acoustic Source Localization," Ultrasonics, 54(1), 25-38 (2014)

[2] T. Kundu, S. Das and K. V. Jata, "Point of Impact Prediction in Isotropic and Anisotropic Plates from the Acoustic Emission Data," Journal of the Acoustical Society of America, 122(4), 2057-2066 (2007).

[3] T. Kundu, S. Das, S. A. Martin and K. V. Jata, "Locating Point of Impact in Anisotropic Fiber Reinforced Composite Plates," Ultrasonics, 48(3), 193-201 (2008).

[4] T. Kundu, S. Das and K. V. Jata, "Impact Point Detection in Stiffened Plates by Acoustic Emission Technique," Smart Materials \& Structures, 18 (2009).

[5] T. Hajzargerbashi, T. Kundu, and S. Bland, "An Improved Algorithm for Detecting Point of Impact in Anisotropic Inhomogeneous Plates," Ultrasonics, 51(3) (2011).

[6] M. Koabaz, T. Hajzargarbashi, T. Kundu, and M. Deschamps, "Locating the Acoustic Source in an Anisotropic Plate," Structural Health Monitoring: An International Journal, 11(3), 315-323 (2012).

[7] H. Nakatani, T. Hajzargarbashi, K. Ito, T. Kundu and N. Takeda "Locating point of impact on an anisotropic cylindrical surface using acoustic beamforming technique," Key Engineering Materials (Special issue on Structural Health Monitoring: Research and Applications), 558, 331-340 (2013).

[8] J. A. Nelder and R. Mead, "A Simplex Method for Function Minimization," Comp. J., 7, 308-315 (1965).

[9] N. A. Barricelli, "Symbiogenetic Evolution Processes Realized by Artificial Methods," Methodos, 143182 (1957).

[10] A. Fraser and D. Burnell, [Computer Models in Genetics], McGraw-Hill, New York (1970).

[11] T. Kundu, "A new technique for acoustic source localization in an anisotropic plate without knowing its material properties," $6^{\text {th }}$ European Workshop on Structural Health Monitoring, Dresden, Germany, July 3-6 (2012)

[12] T. Kundu, H. Nakatani and N. Takeda, "Acoustic source localization in anisotropic plates," Ultrasonics, 52(6), 740-746 (2012).

[13] F. Ciampa and M. Meo, "A New Algorithm for Acoustic Emission Localization and Flexural Group Velocity Determination in Anisotropic Structures," Composites Part A, 41, 1777-1786 (2010).

[14] F. Ciampa, M. Meo and E. Barbieri, "Impact Localization in Composite Structures of Arbitrary Cross Section," Structural Health Monitoring: An International Journal, 11, 643-655 (2012).

[15] M. G. Baxter, R. Pullin, K. M. Holford and S. L. Evans, "Delta T source location for acoustic emission," Mechanical Systems and Signal Processing, 21, 1512-1520 (2007).

[16] D. Xiao, T. He, Q. Pan, X. Liu, J. Wang and Y. Shan, "A novel acoustic emission beamforming method with two uniform linear arrays on plate-like structures," Ultrasonics, 54, 737-745 (2014).

[17] T. Kundu, X. Yang, H. Nakatani and N. Takeda "A two-step hybrid technique for accurately localizing acoustic source in anisotropic structures without knowing their material properties, " Ultrasonics, 56, 271-278 (2015). 